\title{
Multilinguales
}

\section{Les technologies mobiles à l'université algérienne : entre usages et représentations des enseignants}

Mobile technologies at the Algerian university : between uses and

representations of teachers

\section{Sami Redjimi et Billel Ouhaibia}

\section{(2) OpenEdition}

\section{Journals}

Édition électronique

URL : https://journals.openedition.org/multilinguales/5203

DOI : 10.4000/multilinguales.5203

ISSN : 2335-1853

Éditeur

Université Abderrahmane Mira - Bejaia

Référence électronique

Sami Redjimi et Billel Ouhaibia, «Les technologies mobiles à l'université algérienne : entre usages et représentations des enseignants », Multilinguales [En ligne], 13 | 2020, mis en ligne le 15 juillet 2020, consulté le 18 mai 2021. URL : http://journals.openedition.org/multilinguales/5203 ; DOI : https:// doi.org/10.4000/multilinguales.5203

Ce document a été généré automatiquement le 18 mai 2021.

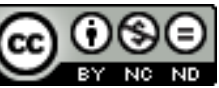

Multilinguales est mise à disposition selon les termes de la Licence Creative Commons Attribution Pas d'Utilisation Commerciale - Pas de Modification 4.0 International 


\title{
Les technologies mobiles à l'université algérienne : entre usages et représentations des enseignants
}

\author{
Mobile technologies at the Algerian university : between uses and \\ representations of teachers
}

Sami Redjimi et Billel Ouhaibia

1 La société actuelle est une société de l'information ayant pour paradigme économique un modèle basé sur le savoir et la connaissance. Dans une telle conjoncture, un rapport étroit s'est créé entre l'information et la technologie. En effet, les technologies de l'information et de la communication (TIC), utilisées pour créer, modifier, analyser et diffuser de l'information, sont devenues un véritable levier de développement économique et social, et conséquemment, une des spécificités phares des sociétés évoluées. Par ailleurs, cela a également fait des TIC une des priorités pour les pays en voie de développement, tel que l'Algérie, puisqu'elles leur permettent de se moderniser et d'entrer de plain-pied dans le monde de l'information.

Outre les champs social, professionnel, économique, faisant appel aux TIC, il est également un terrain des plus cruciaux, celui de l'enseignement/apprentissage.

2 Ainsi, à l'instar de tout autre domaine, les TIC ont été investies dans l'enseignement/ apprentissage afin d'apporter modernité et renouveau à un appareil de formation qui commençait à accuser le poids des décennies et des méthodes désuètes. Le but étant également de développer des compétences transversales chez les apprenants et les amener à construire une culture numérique profitable; d'autant plus que les apprenants de ces deux dernières décennies sont décrits comme des natifs du numérique. Prensky, inventeur du terme angliciste d'origine Digital natives, déclare que: «Nos apprenants ont radicalement changé. Les apprenants d'aujourd'hui ne sont plus les personnes pour lesquelles notre système d'éducation a été créé pour enseigner " 
(2001: 1). En conséquence de quoi, au cours de ces dernières années, les TIC se sont emparées des salles de cours au profit de classes équipées en vidéoprojecteurs, ordinateurs, tablettes, Tableau Blanc Interactif (TBI), microphones, équipements de son, etc.

3 Ceci étant dit, les percées du numérique, notamment dans l'enseignement/ apprentissage, restent limitées dans les pays émergents tel que l'Algérie. De multiples recherches attestent que cela revient en grande partie au manque de financements, aux coûts occasionnés par ces technologies, et à l'absence cruelle de formation de personnel compétent en la matière. C'est pour tenter de résoudre de telles difficultés qu'une nouvelle orientation de recherche sur les TIC dans l'enseignement a vu le jour, celle de l'apprentissage mobile (dans ce qui suit AM).

4 L'objectif de cet article est donc de mieux comprendre le concept de l'AM et de cerner la réflexion qu'y portent les enseignants universitaires algériens. Pour ce faire, nous aborderons, tout d'abord, les notions de base qui caractérisent l'AM. Nous expliciterons, par la suite, les spécificités de la recherche par questionnaire que nous avons faite auprès d'enseignants universitaires. Nous terminerons, enfin, par la présentation et la discussion des résultats obtenus.

\section{L'apprentissage mobile, notions de base}

5 Si les ordinateurs et les infrastructures TIC (ordinateurs, réseau interconnecté, câblage pour la connexion internet, etc.) sont compliqués à acquérir, à mettre en place et à entretenir, les outils mobiles tels que les smartphones et tablettes ont le vent en poupe grâce à une grande démocratisation due à leur développement, à leur praticité et mobilité, à une concurrence poussée sur les marchés et des prix de plus en plus accessibles. Avec de telles caractéristiques, l'adoption de ce type d'outils dans l'enseignement/apprentissage a toutes les chances de réussite.

D'un point de vue historique, l'emploi des outils mobiles et de surcroit personnels dans des tâches de travail a débuté, d'abord, en contexte d'entreprise il y'a plus ou moins une quinzaine d'années. Les ordinateurs de bureau et autres équipements dispendieux et rapidement obsolètes ont laissé place aux téléphones intelligents, ordinateurs portables et autres tablettes numériques. Ces artefacts sont devenus petit à petit très exploités, aussi bien des professionnels d'entreprise, que, par la suite, des enseignants et apprenants dans et en dehors de la classe. Réfèrent à cette modalité plusieurs dénominations: BYOD pour l'anglais "Bring Your Own Device", ou encore BYOT «Bring Your Own Technology» et CYOD «Choose Your Own Device»; AVAN en français pour "Apportez Vos Appareils Numériques», ou encore PAP «Prenez vos Appareils Personnels», et AVEC «Apportez Votre Equipement Personnel de Communication ».

A l'instar des entreprises ; les écoles, les universités et les institutions de formation ont vu dans le BYOD un moyen simple de favoriser l'enseignement/apprentissage par les technologies à moindres coûts. Outre cet avantage, le BYOD permet aux apprenants de choisir et d'apporter l'outil qui leur sied le plus afin d'en faire usage en classe (ordinateur portable, smartphone, tablette). L'intérêt étant également que les acteurs enseignants et apprenants utilisent des outils dont ils ont l'habitude et qu'ils maitrisent parfaitement bien. 
A ce titre, l'UNESCO définit l'AM ainsi :

L'apprentissage mobile fait appel à la technologie mobile, seule ou combinée à d'autres technologies de l'information et de la communication (TIC), pour permettre d'apprendre en tout lieu et à tout moment. L'apprentissage prend alors les formes les plus diverses : on peut utiliser les appareils portables pour accéder aux ressources éducatives, se connecter aux autres ou créer du contenu, aussi bien à l'intérieur qu'à l'extérieur de la salle de classe. L'apprentissage mobile s'applique aussi aux objectifs éducatifs au sens large, qu'il s'agisse de mieux gérer les systèmes scolaires ou d'améliorer la communication entre les écoles et les familles (2013:06).

L'on comprend à partir de cette définition que les outils nomades possèdent une pléthore de fonctionnalités qui peuvent être mises en œuvre dans diverses configurations, dans et en dehors de la salle de classe : recherche d'informations et de contenus éducatifs, partage de ressources avec les pairs, enregistrements audios et vidéos, stockage de données, collaboration avec les pairs sur des forums et plateformes pédagogiques, etc. En somme, la force de ces appareils réside dans leur accessibilité, facilité d'usage, transportabilité, diversité et évolution, multifonctionnalité, et surtout leur omniprésence. En outre, ces fonctionnalités et caractéristiques (des outils mobiles) donnent la possibilité à l'enseignant/formateur de proposer à son public des contenus et des activités plus riches, ludiques et interactives; et ce, même si l'institution à laquelle il appartient n'a ni l'équipement, ni le budget, ni l'intention pour s'inscrire dans ce genre de perspectives.

8 Le Vincent et Botino (2017) établissent trois (03) situations distinctes d'intégration du BYOD dans l'enseignement. La première est une initiative d'un enseignant isolé, qui, par motivation, instaure ce genre d'expérience dans sa classe, parfois sans même prévenir le chef d'établissement. Le partage d'expérience avec ses collègues peut amener ces derniers à tenter la démarche à leur tour. La deuxième est une volonté de l'établissement ou de l'institution de mettre en place une approche BYOD en y invitant les enseignants et en leur proposant des formations techniques sur les usages pédagogiques des outils mobiles. L'établissement met également à la disposition des enseignants et apprenants un réseau WI-FI accessible en classe. La troisième s'apparente plus à une situation planifiée et contrôlée par l'établissement, les enseignants, les équipes techniques, les intervenants extérieurs (par exemple les parents). Ici, le BYOD devient un projet pédagogique dans lequel les enseignants sont formés et reçoivent une aide technique pour les accompagner lors des cours. Le projet est surveillé et les progrès et les impacts évalués.

Le BYOD étant un champ de recherche relativement récent, il y'a peu, voire quasiment pas, de modèles théoriques d'intégration qui sont attestés et approuvés par la communauté scientifique. Ceci étant dit, certains d'entre eux émergent petit à petit, à l'exemple de celui de Fiévez et Dumouchel (2015). 
Figure 1 : Modèle d'intégration du BYOD (Fiévez \& Dumouchel, 2015)

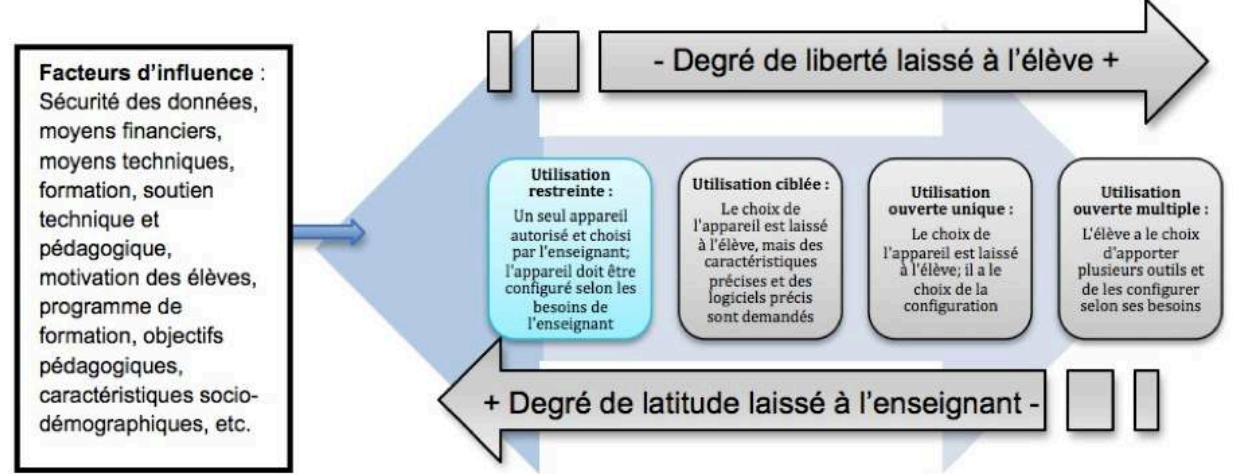

10 Dans leur modèle, ces auteurs différencient quatre (04) formules d'intégration du BYOD tributaires du niveau de liberté qu'octroie l'enseignant aux apprenants dans le choix de leur matériel technologique pour une utilisation pédagogique en classe. Les formules sont les suivantes :

- Une utilisation restreinte où l'enseignant est le maitre à bord, il choisit le type d'appareil à utiliser, les applications à avoir, les ressources à consulter, etc. L'inconvénient est que ces aspects sont imposés aux apprenants ;

- Une utilisation ciblée où l'enseignant laisse le choix aux apprenants de choisir un appareil, mais qui doit obéir à certaines caractéristiques (capacité du processeur, taille de l'écran, mémoire vive, etc.). Toute la latitude reste du côté enseignant ;

- Une utilisation ouverte unique où l'apprenant se voit avoir le choix de son appareil et ses applications. L'enseignant doit se montrer flexible et s'adapter aux apprenants ;

- Une utilisation ouverte multiple où une liberté totale est accordée aux apprenants qui peuvent choisir un ou plusieurs appareils et les applications qu'ils souhaitent utiliser. Si la situation se complexifier davantage ici pour l'enseignant, les possibilités d'innovation pédagogique s'en trouvent décuplées. Cette dernière formule recouvre toutes les potentialités du BYOD.

11 Fiévez et Dumouchel (2015) signalent également l'influence que peuvent avoir des facteurs extérieurs sur les environnements BYOD. Ces facteurs se rapportent aux moyens financiers, à la formation et au soutien technique, à la motivation des apprenants, à la sécurité des données, aux objectifs pédagogiques et aux caractéristiques sociodémographiques, etc. Selon ces mêmes auteurs, des adaptations structurelles et comportementales sont nécessaires au préalable de toute initiative BYOD. A titre d'exemples, ils recommandent aux institutions scolaires et universitaires de façonner une installation internet permettant de nombreux accès en simultané (tous les apprenants accèdent au réseau WIFI en même temps); de fournir un soutien technique aux enseignants en tout temps; de responsabiliser les apprenants pour une stricte utilisation pédagogique des outils numériques en classe (au lieu de les menacer). A cela, Fiévez \& Dumouchel (2015) ajoutent que si l'enseignant doit choisir les activités à entreprendre avec les outils mobiles, il n'est pas obligatoire pour les apprenants de recourir aux mêmes logiciels et ressources. Au contraire, une diversification dans la manière d'opérer ne peut qu'être bénéfique pour un apprentissage varié.

12 A ce propos, Le Vincent et Botino (2017), en guise de bilan d'une expérience BYOD, recensent cinq (05) emplois des outils nomades :

- Ressource ponctuelle : dictionnaire, calculatrice, conjugueur...; 
- Cahier d'exercices: sous forme d'applications pédagogiques incluant des exercices d'entrainement. Ces dernières peuvent aussi fournir des informations du style: le temps passé sur les activités, le nombre et le type d'erreur. Des exercices ciblés peuvent être proposés en remédiation (personnalisation de l'apprentissage, différenciation et autonomie) ;

- Point d'accès à des supports multimédia : Des vidéos, des audios, des conférences, des podcasts, des visioconférences enregistrées, etc. Ces ressources peuvent être visionnées en tout temps et n'importe où ;

- Cahier journalier : Administrativement parlant, l'accès pour un enseignant aux feuilles d'absence et aux feuilles de notes fournies par l'administration sur un espace de partage et de stockage des documents administratifs ;

- Fiche de suivi des apprentissages: Certaines applications mobiles permettent aux enseignants de faire une brève évaluation en direct (en classe), sous forme de questionnaires en ligne, par exemple. L'enseignant peut accéder instantanément à une analyse des résultats et évaluer le degré de compréhension des élèves.

Par ailleurs, le BYOD n'est pas non plus une panacée à tous les maux des TIC dans l'enseignement. Lui aussi, à l'instar de toutes les autres approches, se caractérise par des avantages et des inconvénients.

En somme, les avantages du BYOD se rapportent à la maitrise parfaite des apprenants et des enseignants de leurs outils numériques, à l'accès à tout type d'informations et supports, à une disponibilité à tout moment, à la personnalisation des leçons, à une économie de dépenses pour les institutions, à la facilité de partage et de collaboration entre apprenants et enseignants, à une meilleure motivation des apprenants. Sur ce dernier point, Roux Bellicaud (2014) explique que c'est le lien affectif entretenu avec l'appareil (surtout le smartphone) qui ravit les apprenants lors de son utilisation dans un cadre pédagogique. Les inconvénients, eux, se rapportent à une complexification du rôle de l'enseignant de par la multiplicité des ressources et des outils à gérer (et qu'il ne maitrise pas toujours), à un souci d'équité entre les apprenants quant aux appareils (modernité, qualité, options, etc.), à un éventuel conflit avec les parents si ces derniers décident de ne pas laisser leurs enfants utiliser les appareils mobiles afin de les protéger d'une manière ou d'une autre. Ceci dit, rappelons que la plupart de ces inconvénients ne se posent qu'avec un jeune public (enfants) et non avec un public adulte.

De manière globale, le BYOD présente un potentiel pédagogique non négligeable pour les institutions scolaires et universitaires, à plus forte raison lorsqu'il s'agit des pays en voie de développement, puisque c'est eux qui rencontrent le plus de difficultés quant à une incorporation efficace des TIC. L'intérêt de la présente étude est de cerner les représentations et les avis des enseignants universitaires algériens quant à une éventuelle mise en place d'une approche BYOD à l'université. Nous en profiterons pour sonder les utilisations qu'ils font de leurs outils nomades dans un contexte privé et professionnel.

\section{Matériel et méthode de l'enquête}

A l'instar de tout autre domaine, en matière de technologies, les conceptions et les représentations des individus impactent de manière directe leurs comportements et usages. Dans cette perspective, nous nous sommes intéressés aux opinions et avis des 
enseignants universitaires sur les outils mobiles et leur éventuel emploi en classe de langue. Pour ce faire, nous avons eu recours à la méthode du questionnaire. Nous avons donc administré un questionnaire à des enseignants universitaires appartenant au département de français de l'université d'Annaba. Trente-deux (32) réponses ont été recueillies sur un total d'une soixantaine d'enseignants du même département. Cela nous permet d'atteindre un niveau de représentativité satisfaisant quant à ce public en particulier.

16 Le questionnaire en lui-même se compose de deux parties : la première porte sur des informations générales du type sexe, âge, ancienneté, grade ; la deuxième comporte principalement des questions à choix multiples accompagnées de justifications pour certaines d'entre elles. Il est à noter que la confection de notre questionnaire s'est inspirée du travail fait par Bacha, Ben Abid-Zarrouk, Kadi, et Mabrour (2016) qui s'est intéressé de près aux TIC dans les universités du Maghreb. La diffusion du questionnaire et la récolte des réponses se sont faites entre mai et août 2019. Des analyses qualitatives et quantitatives ont été faites sur les réponses obtenues. Ainsi qu'il a été explicité plus haut, cela nous renseigne, d'une part, sur les outils auxquels recourent les enseignants, le plus souvent, dans la sphère privée et dans la sphère professionnelle (préparation des cours, partage des ressources, etc.), et d'autre part, sur leurs avis quant à une utilisation des outils mobiles en classe.

\section{Résultats et discussion}

Dans ce qui suit, nous présentons les résultats de notre enquête en essayant de les discuter et de les interpréter :

S'agissant des caractéristiques des enseignants-participants à l'enquête, nous observons (graphique 1) que la majorité en est féminine (78.1\% de femmes VS $21.9 \%$ d'hommes).

\section{Graphique 1 : Sexe}

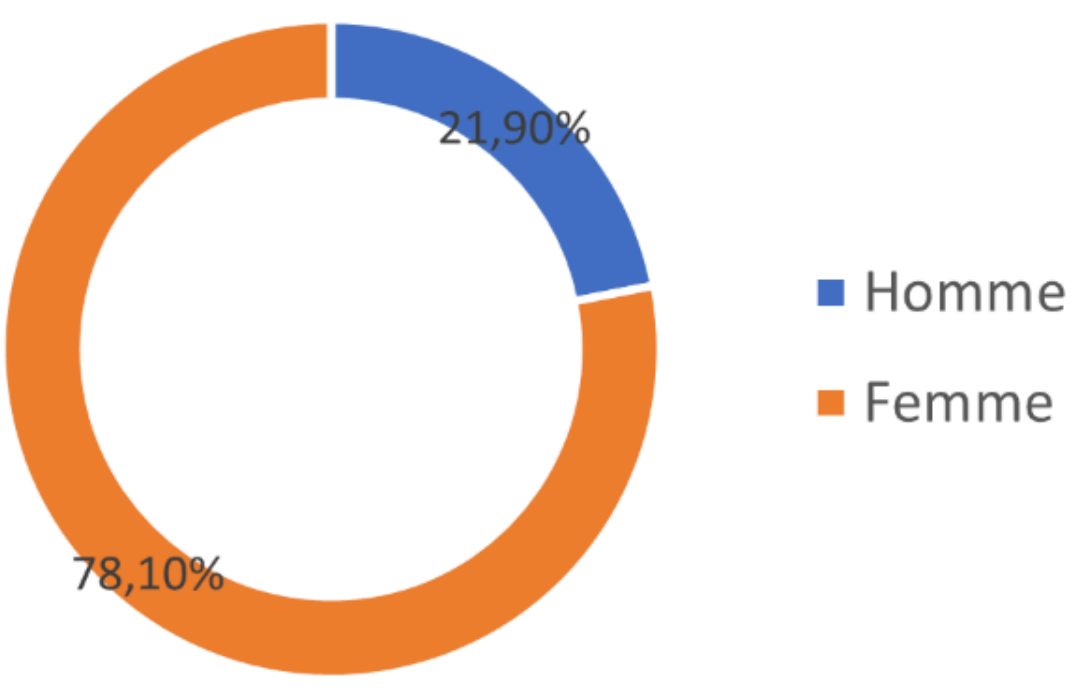

Le graphique 2 montre une hétérogénéité quant aux âges des enseignants. En effet, ces derniers varient de moins de 30 ans à plus de 50 ans. 


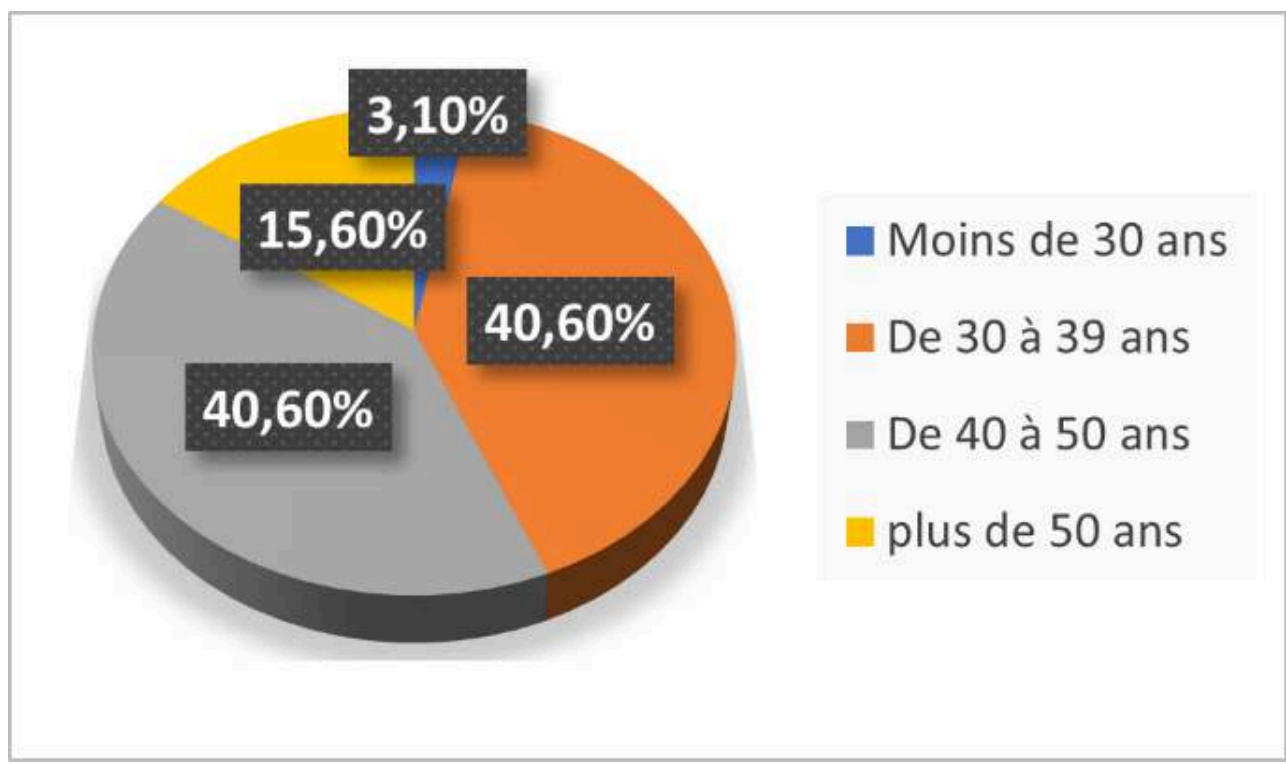

Le graphique 3 met en avant l'aspect de l'ancienneté des enseignants dans l'enseignement universitaire. A ce titre, nous observons que la majorité d'entre eux enseignent depuis 5 à 15 ans; une minorité a moins de 5 ans d'expérience.

Graphique 3 : Ancienneté dans l'enseignement

\section{Moins de 5 ans $\square$ De 5 à 15 ans $\square$ Plus de 15 ans}

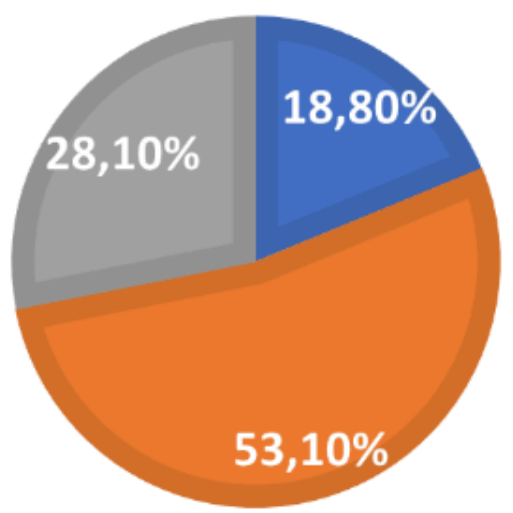

Le graphique 4 ci-dessous distingue le grade des enseignants universitaires ayant participé à notre enquête. La grande majorité d'entre eux ont un grade de maitreassistant $\mathrm{A}$, suivi par la deuxième portion ayant un grade de maitre-assistant $\mathrm{B}$. 
Graphique 4 : Grade des enseignants

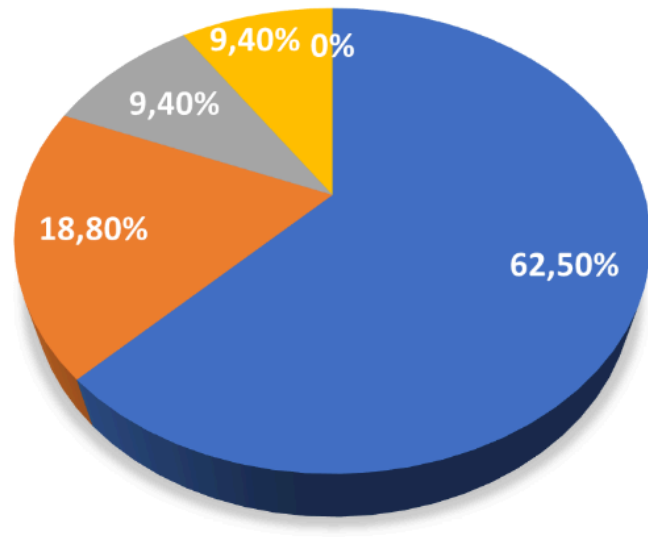

Maitre assistant A

Maitre assistant B

Maitre de conférences $\mathrm{A}$

Maitre de conférences B

Professeur

18 Les caractéristiques du public enseignant de notre enquête ayant été explicitées, nous examinons dans ce qui suit leurs utilisations des outils mobiles et leurs avis sur l'introduction de ces outils en classe de langue. Certaines données feront l'objet d'un traitement croisé (entre les questions).

19 Nous avons souhaité savoir si les enseignants possèdent l'un des appareils mobiles ou plusieurs d'entre eux. Les réponses (graphique 5) mettent en avant que l'ordinateur portable reste l'outil le plus possédé avec $100 \%$ des réponses. Le smartphone lui, est à la deuxième place avec un taux de possession de $81.3 \%$; devant la tablette numérique, en troisième place, avec un taux de possession de seulement $37.5 \%$. 
Tablette numérique

\section{Ordinateur portable}

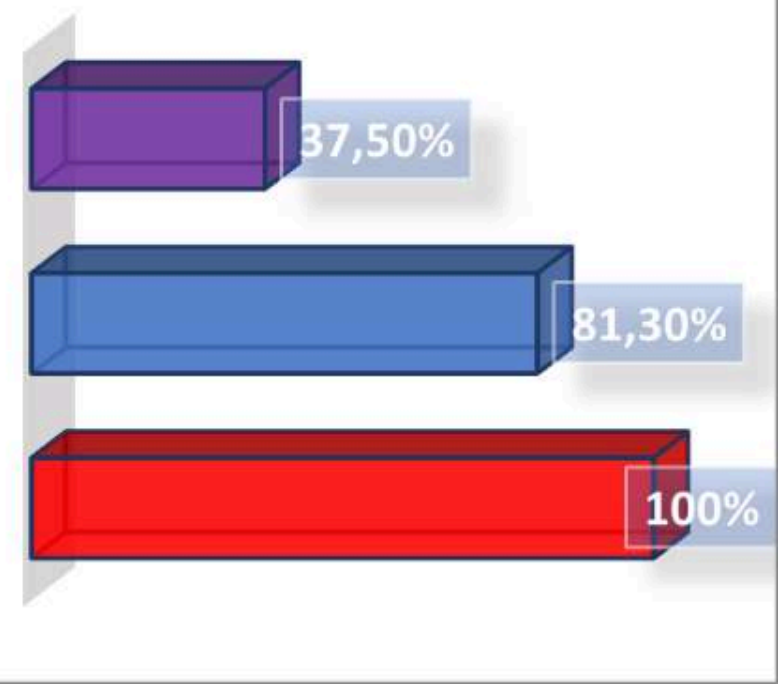

Ces taux de possession peuvent être expliqués par le fait que l'ordinateur portable reste l'outil incontournable de travail et de loisirs (films, musique, etc.). La tablette numérique tend à être de moins en moins achetée au profit des smartphones à grand écran (aussi appelés phablettes) qui sont plus ergonomiques et pratiques avec des performances équivalentes, si ce n'est plus. Le smartphone reste un outil multitâche incomparable (communication, internet, réseaux sociaux, lecture, documents, appareil photo, enregistreur vocal, etc.).

Le graphique 6 montre les résultats de la question Quel appareil utilisez-vous le plus dans un cadre personnel? croisés avec la variable de l'âge. De prime abord, il est à observer que seuls l'ordinateur portable et le smartphone sont utilisés. Ainsi, ces réponses font écho aux résultats de la précédente question et les confirment par la même occasion. De même, nous distinguons que les enseignants les plus jeunes sont ceux qui recourent le plus au smartphone dans le cadre privé. A contrario, les enseignants quelque peu plus âgés recourent à l'ordinateur portable. 
Graphique 6 : L'appareil le plus utilisé en fonction de l'âge

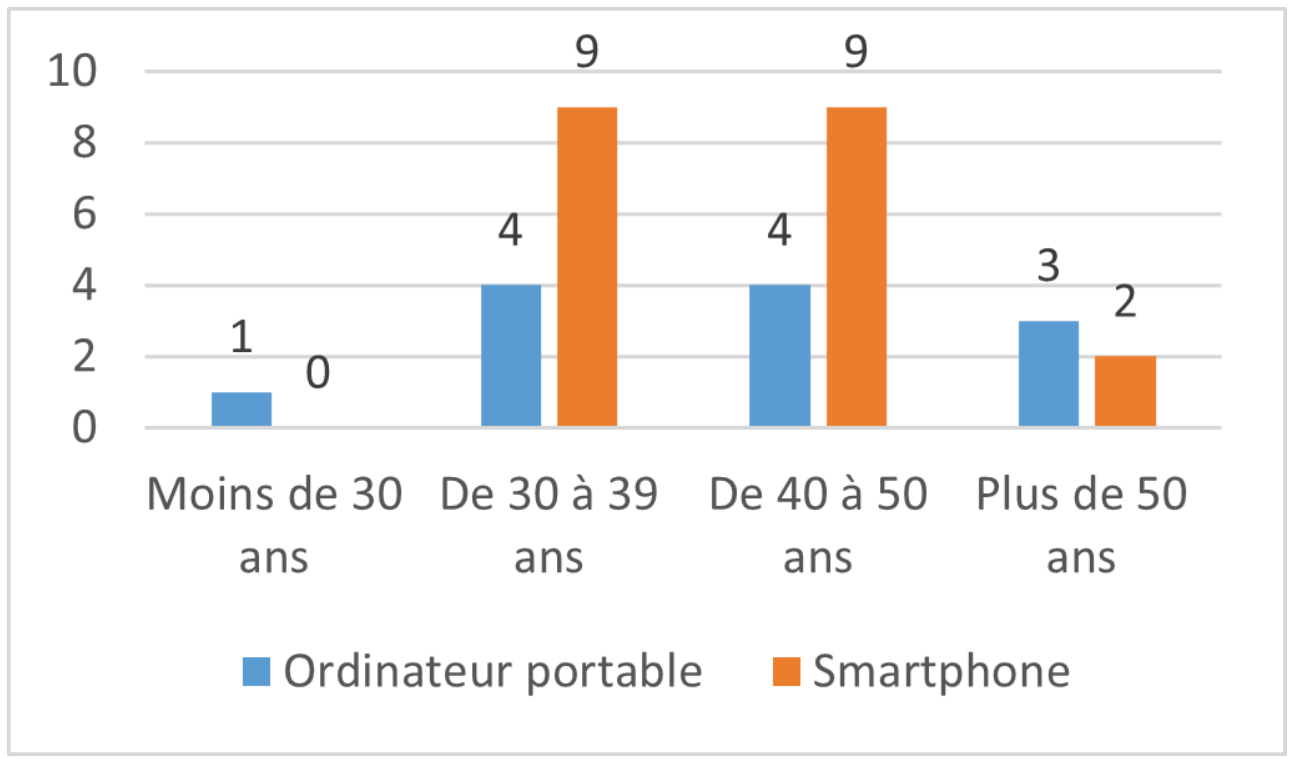

Les justifications avancées par les enseignants ayant répondu en faveur du smartphone tournent autour de la praticité, la légèreté, la facilité et la maniabilité, la transportabilité, la disponibilité, le multitâche. Les enseignants ayant répondu en faveur de l'ordinateur avancent des raisons telles que la grandeur de l'écran et du clavier, le confort dans le travail sur les documents (Word, Excel, etc.), le côté professionnel de l'outil.

Dans la même perspective, nous avons cherché à savoir quel est l'appareil le plus privilégié pour un accès à internet. A l'instar de la question précédente, nous avons croisé ces données avec celles de l'âge des enseignants. Le graphique 7 laisse paraitre que le smartphone reste l'outil le plus employé pour accéder à internet chez la tranche d'âge des 30 à 50 ans. En ce qui concerne les enseignants ayant plus de 50 ans, les résultats laissent entrevoir qu'ils s'attachent plutôt à l'ordinateur. Même si la participation de cette tranche d'âge reste légère (15.6\%), un comportement global est facile à déceler par des résultats univoques (tous les enseignants de plus de 50 ans ont répondu qu'ils n'utilisaient que l'ordinateur pour accéder à internet à l'exception d'un seul qui a répondu la tablette numérique). 


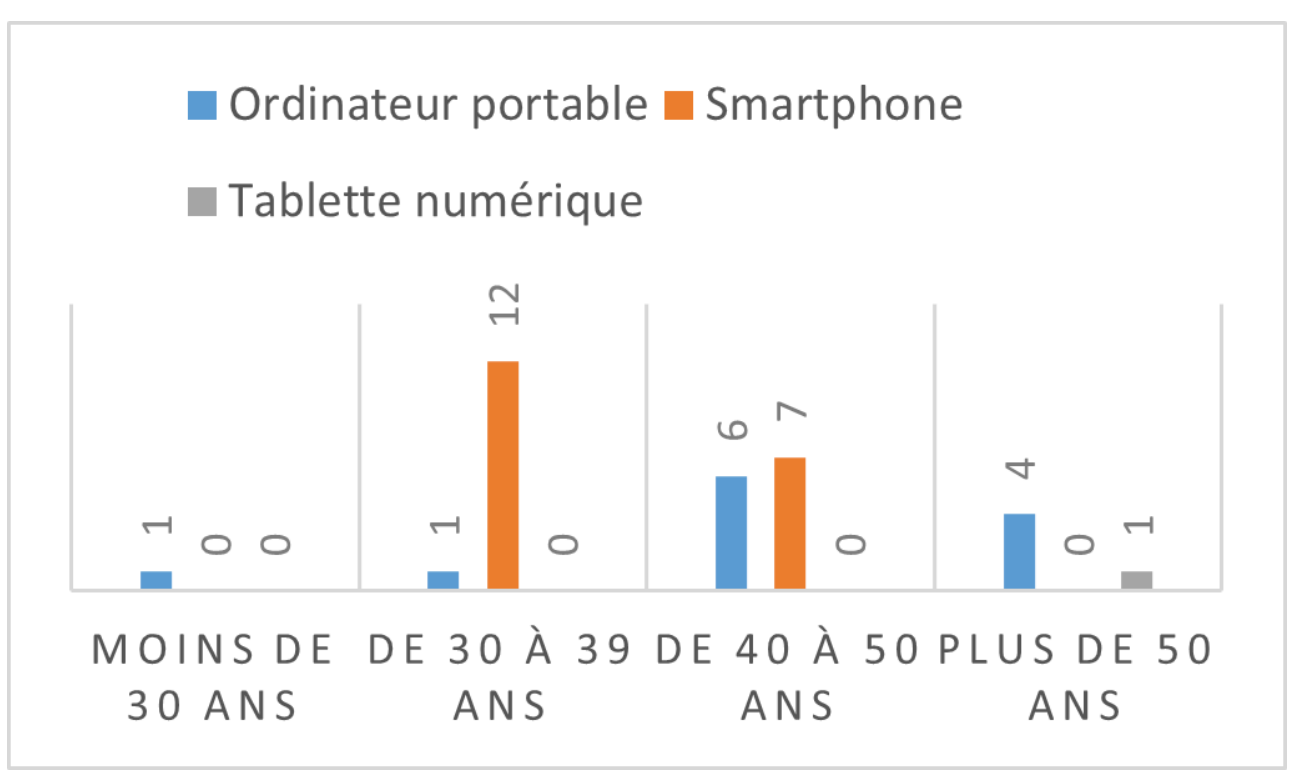

Ce fait peut s'expliquer par l'idée que les enseignants relativement jeunes ont pris le train du développement de la technologie mobile en marche. Désormais, ils utilisent le smartphone de manière plus importante et presque similaire à la génération native du numérique (envoyer et recevoir des mails, télécharger des documents de travail, partager et communiquer grâce aux réseaux sociaux, etc.). Les enseignants les plus âgés recourent à l'ordinateur portable sans doute à cause de son grand écran (éventuels problèmes de vue), d'un manque de maitrise des smartphones, d'une meilleure familiarité avec l'ordinateur (qui d'ailleurs existait bien avant le smartphone).

Dans ce cheminement, nous avons souhaité savoir quel outil utilisaient les enseignants dans le cadre de la préparation et dispensation des cours (les enseignants avaient la possibilité de cocher plusieurs réponses ici). Le graphique 8 dénote sans la moindre surprise une utilisation importante de l'ordinateur portable avec un taux de $96.9 \%$. Vient ensuite le smartphone avec un usage de $18.8 \%$, et en enfin la tablette numérique avec seulement $6.3 \%$. 


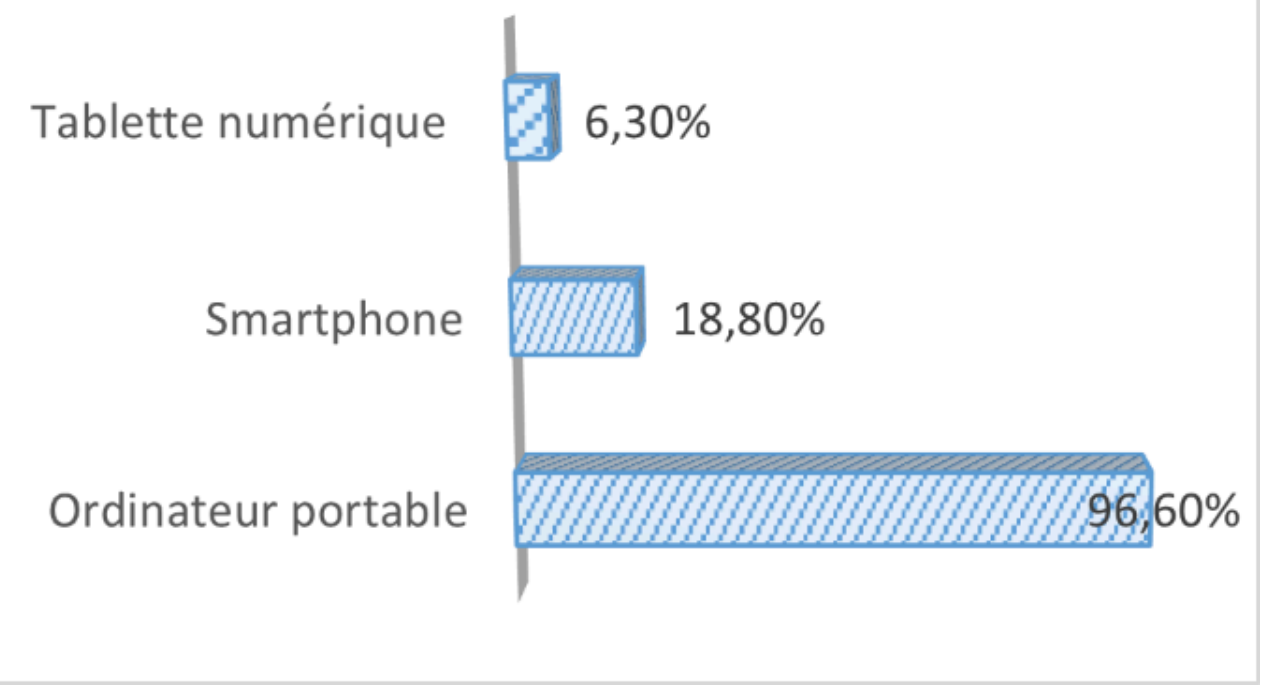

La dominance de l'ordinateur portable dans le cadre professionnel de préparation des cours n'est en rien étonnante. En effet, l'ordinateur reste l'outil le plus efficace pour mener plusieurs tâches en même temps (recherche d'information, rédaction d'un cours ou d'un syllabus, envoi de mails, etc.). Son grand écran, son clavier, sa souris, son architecture des onglets facilitent la prise en charge et le traitement de plusieurs documents de différents types au même moment.

En outre, nous nous sommes demandé si les enseignants encourageaient leurs étudiants à utiliser les appareils mobiles à écrans tactiles (smartphones, tablettes) dans le cadre des cours et au sein même de la classe. 75 \% des enseignants ayant participé à l'enquête affirment que oui, contre $25 \%$ qui attestent que non. Le graphique 9 présente le croisement de ces données avec la variable du grade des enseignants. Nous observons que ce n'est pas les enseignants les plus expérimentés, et qui maitrisent relativement mieux les diverses méthodes d'enseignement, qui poussent les étudiants vers l'AM et par ricochet l'autonomie. En effet, ce sont les jeunes enseignants ayant majoritairement un grade de maitre-assistant A qui déclarent encourager les étudiants en ce sens. 
Graphique 9 : L'encouragement de l'utilisation des outils mobiles à écran tactile en classe selon le grade

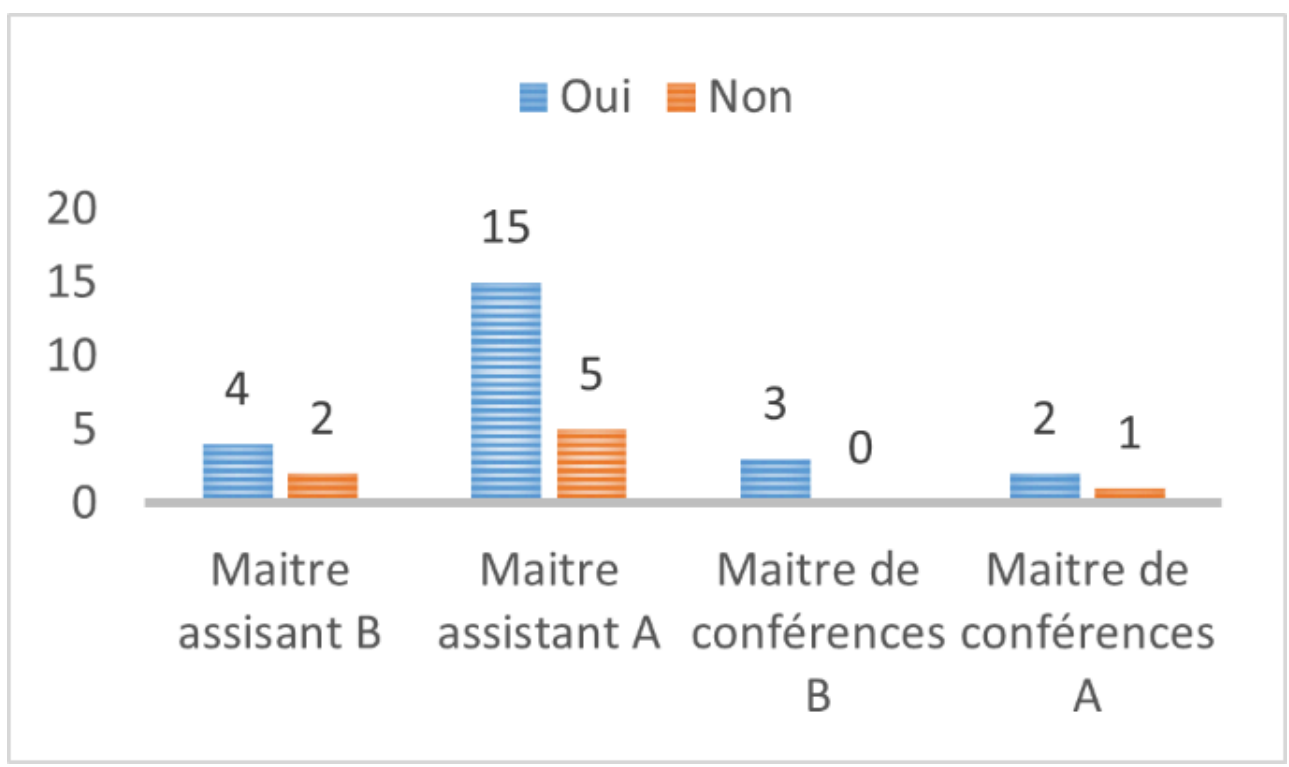

Le fait que ce soit les jeunes enseignants maitres-assistants qui encouragent leurs étudiants à recourir aux smartphones et tablettes durant le cours peut être expliqué par le fait que c'est justement ces enseignants qui sont les plus au fait de l'évolution des méthodes pédagogiques et des dernières nouveautés en matière d'approches innovantes, et ce en partie grâce à leurs recherches doctorales toujours en cours. Parfois même, l'innovation qu'ils essaient d'engendrer en classe fait partie d'une expérimentation scientifique. Ajoutons à cela que c'est ces enseignants relativement jeunes qui utilisent et maitrisent de manière satisfaisante ces outils (comparativement aux plus âgés). C'est donc cette catégorie d'enseignants qui est la plus prédisposée aux technologies mobiles en classe. Ces enseignants invitent d'ailleurs leurs étudiants à exploiter leurs smartphones afin de rechercher de l'information sur le web, consulter un dictionnaire, lire un document directement sur l'appareil au lieu de l'imprimer, s'enregistrer à l'oral pour une autoscopie, etc.

S'agissant des enseignants ayant répondu défavorablement à un usage des appareils mobiles à écran tactile en classe, ils justifient ce choix par la volonté d'éviter la déconcentration des étudiants et l'usage personnel des appareils, la perte de temps, l'absence d'un réseau WIFI et le manque cruel de formations à l'usage des technologies en classe. Dans cet ordre d'idées, nous avons demandé aux enseignants de classer par ordre d'importance les trois outils mobiles (ordinateur portable, smartphone, tablette) pour une éventuelle intégration en classe dans une visée didactique et pédagogique. Le graphique 10 montre que le smartphone est l'outil privilégié dans ce cas de figure puisqu'il occupe la première place avec 16 voix. Vient ensuite, à la deuxième place, la tablette numérique avec 18 voix, et enfin, l'ordinateur en troisième place avec 14 voix. 
Graphique 10 : Classement des appareils mobiles par ordre d'importance pour une intégration pédagogique en classe

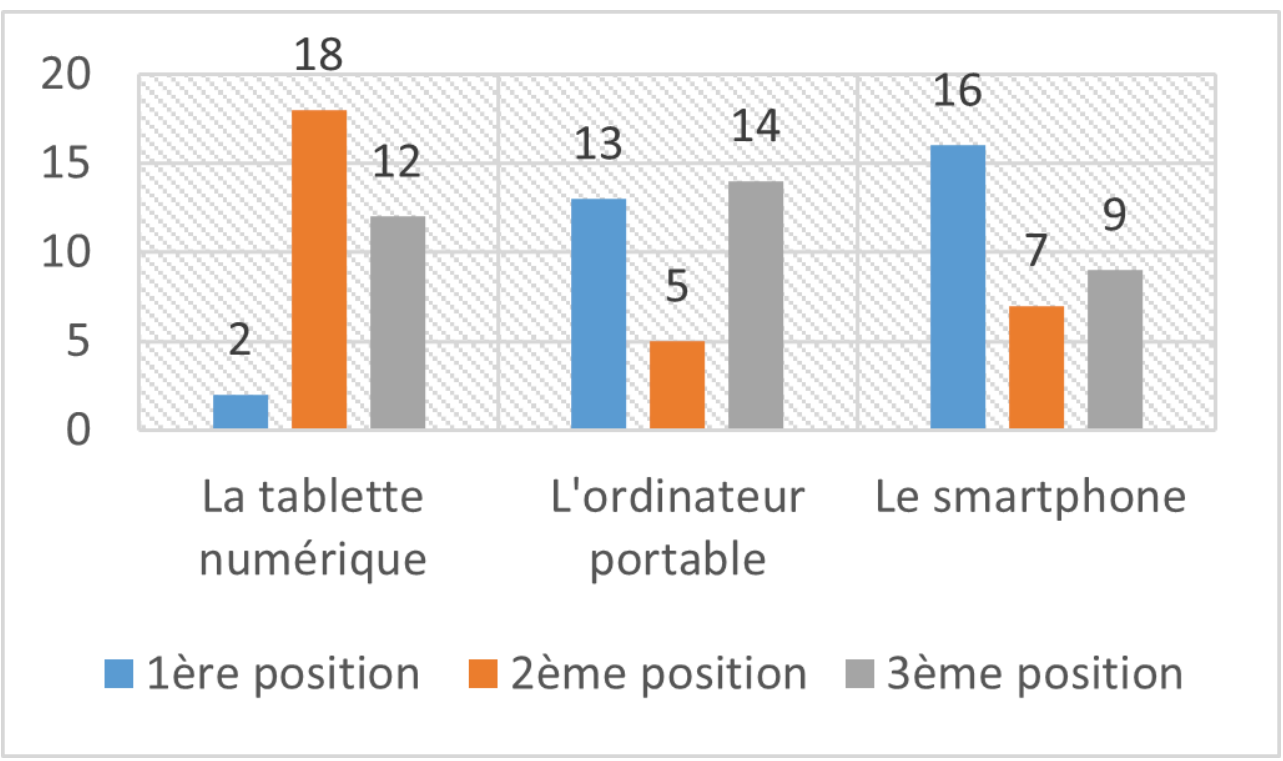

L'occupation de la première place par le smartphone s'explique par ses spécificités de facilité d'usage, de disponibilité, de performances, et de multiusage. La tablette numérique, elle, est à la deuxième place grâce à son écran plus grand et donc à une meilleure lisibilité, mais aussi à cause de son manque de popularité comparé au smartphone. De manière générale, le classement prioritaire de ces deux appareils par les enseignants met l'accent, encore une fois, sur la pertinence du recours à ces outils dans un contexte pédagogique où les TIC sont quasi-inexistantes . L'ordinateur portable quant à lui est à la dernière place puisque, si ce dernier reste l'outil professionnel phare, il n'en demeure pas moins lourd et plus complexe à transporter, surtout si c'est fait de manière quotidienne ou presque.

Si ces questions restent plus ou moins détaillées, nous avons aussi tenu à connaitre, de manière générale, les avis et les représentations quant à l'utilisation de ces appareils nomades en classe. Les possibilités de réponses données allaient de très intéressant à pas du tout intéressant. Nous constatons à partir du graphique 11 qu'une majorité (53.1\%) d'enseignants trouvent l'idée très intéressante, une grande partie la trouve simplement intéressante $(31.3 \%)$, une minorité $(12.5 \%)$, quatre personnes, la trouve peu intéressante, et seule une personne $(3.1 \%)$ ne la trouve pas du tout intéressante. 


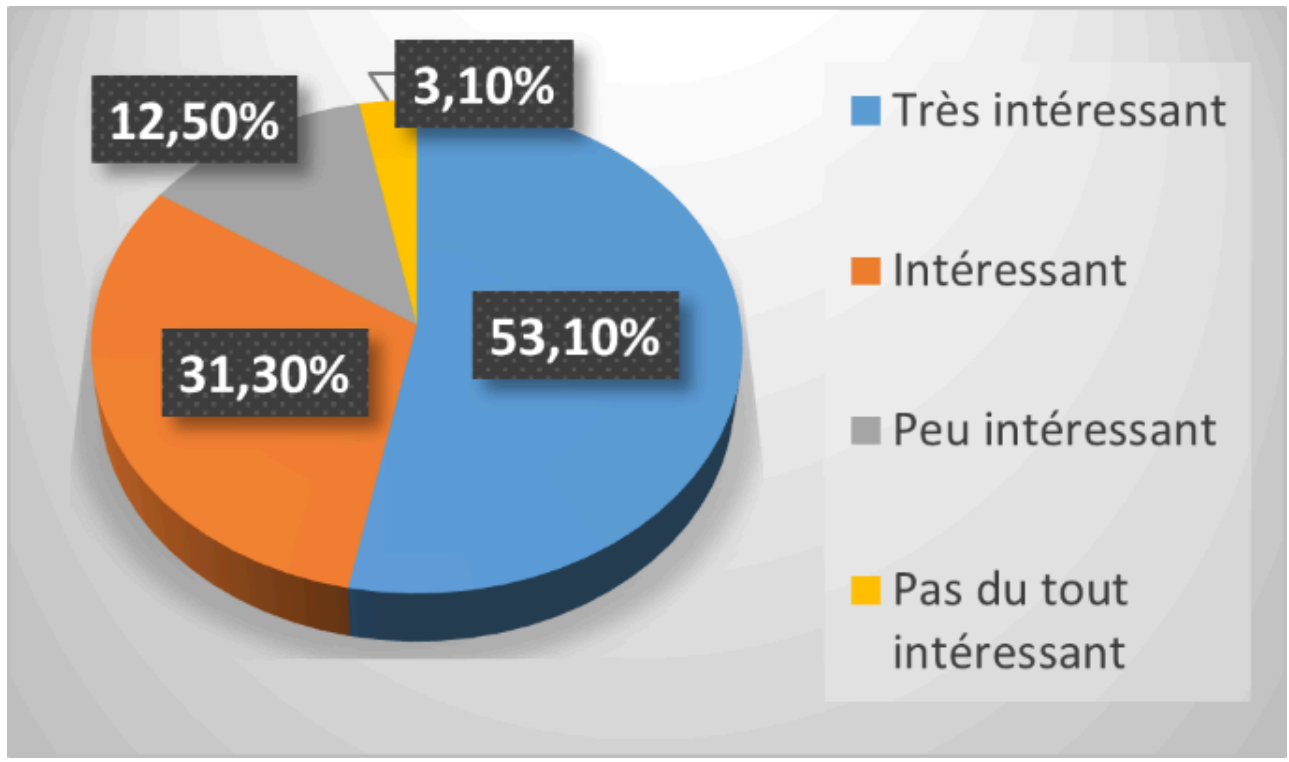

Face à l'innovation et aux possibilités pédagogiques et didactiques que laisse percevoir l'AM, la majorité des sondés affirme être très intéressée ou simplement intéressée par une éventuelle mise en œuvre à l'université. La minorité trouvant le concept peu voire pas du tout intéressant, pense cela, sans doute, à cause des quelques points faibles de l'approche, notamment la déconcentration des étudiants et le possible usage personnel des outils, etc.

La question suivante fait justement écho à celle ci-dessus en demandant aux enseignants s'ils seraient intéressés par une formation portant sur l'usage des appareils mobiles à des fins pédagogiques. Encore une fois, une majorité d'entre eux (84.4\%), soit 27 personnes, dit être intéressée. Seulement $15.6 \%$, soit 5 personnes, disent que non (graphique 12).

Graphique 12 : Intérêt ou non des enseignants quant à une formation à l'apprentissage mobile

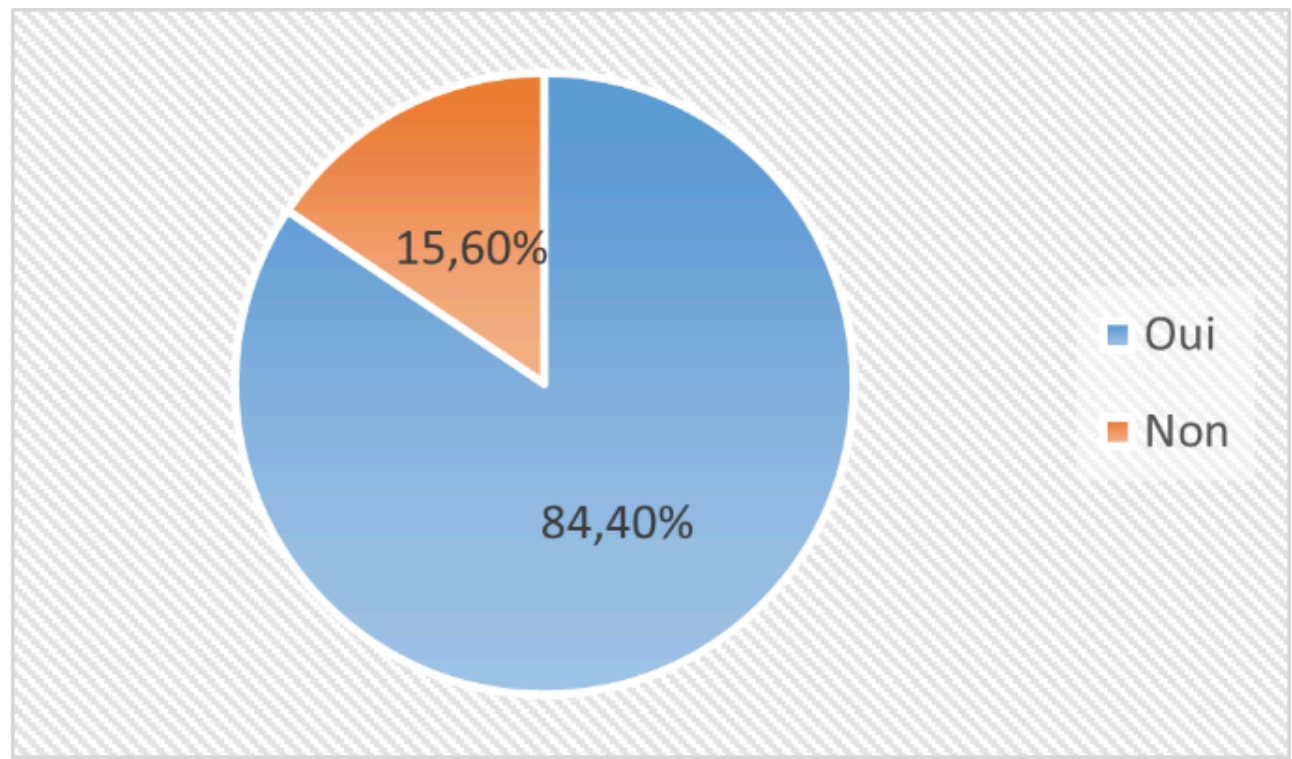


La majorité enseignante a très bien compris qu'elle ne pouvait plus rester insensible face au développement du numérique, à son intégration à tous les domaines de vie et à la considération de sa maitrise comme une compétence indispensable au $21^{\mathrm{e}}$ siècle, à fortiori lorsqu'elle a affaire à des générations natives du numérique et hyperconnectées. Cela témoigne aussi du désir des enseignants d'actualiser leurs méthodes d'enseignement afin d'être à la page. A ce titre, ils justifient leur intérêt par le fait de chercher à être plus performants dans leurs enseignements, d'accéder et de partager l'information rapidement, de découvrir de nouveaux outils et méthodes, de vouloir combler leurs lacunes en matière de technologies, d'être plus créatif et efficace, de pouvoir impliquer l'apprenant dans son apprentissage (recherches, apprendre à apprendre), de vouloir diversifier les pratiques pédagogiques.

S'agissant des enseignants ayant un point de vue opposé, donc défavorable, ils restent attachés à des pratiques d'enseignement classiques. En effet, par manque d'informations ou d'intérêt, ils désapprouvent l'usage de telles technologies dans l'enseignement et penchent plus en faveur des livres. De même, certains d'entre eux disent ne pas être intéressés par une telle formation, soit parce qu'ils l'ont déjà faite, soit parce qu'ils estiment avoir une maîtrise des TIC déjà bien suffisante. Ainsi, il est à remarquer que si le manque de développement et de financement des infrastructures TIC joue un rôle important dans leur absence des paysages scolaire et universitaire algériens, les représentations qu'en ont les acteurs enseignants sont toutefois assez positives. En effet, nous remarquons à partir des résultats exposés ci-dessus, que ce sont les enseignants les plus jeunes et en début de parcours de recherche et d'enseignement qui restent les plus ouverts aux outils nomades dans l'enseignement, et qui les utilisent eux-mêmes dans leur travail et vie quotidienne.

Les technologies de l'information et de la communication représentent une chance et un moyen d'innovation et de renouvellement des pratiques pédagogiques et des modalités de l'enseignement/apprentissage. Nonobstant, leur incorporation aux institutions de formation peut s'avérer problématique du point de vue de la maitrise, $\mathrm{du}$ financement, de la mise en place et de la maintenance. Ce sont justement les difficultés que rencontrent les organismes scolaires et universitaires en Algérie, à l'instar d'autres pays en voie de développement. Face à cela, l'approche de l'AM gagne de plus en plus de terrain pour justement essayer de remédier à ces obstacles et de faire entrer, tant que faire se peut, les technologies au service de l'enseignement/ apprentissage.

Les résultats de notre enquête par questionnaires auprès d'enseignants universitaires à ce sujet dévoilent qu'une majorité d'entre eux reste encline et intéressée par la mise en œuvre d'une telle approche en classe de FLE. A ce titre, nous avons vu que même si l'ordinateur portable reste l'outil de travail privilégié pour un grand nombre d'enseignants, le smartphone, lui, est l'outil de choix pour un éventuel usage des technologies mobiles en classe. Cela en raison de l'absence d'infrastructure TIC au sein des universités algériennes et des caractéristiques de l'outil facilitant son usage et déplacement (interactif, connecté au web, léger et petit, intelligent, multitudes d'options et d'applications, etc.). 


\section{BIBLIOGRAPHIE}

BACHA, J., et al., « Penser les TIC dans les universités du Maghreb : TIC et enseignement/ apprentissage du et en français en contexte universitaire maghrébin », Paris : L'Harmattan, 2016, $331 P$.

BENABED, F., «Intégration des TIC dans l'enseignement au Maghreb : Le discours officiel en Algérie », dans MURIEL FRISCH (dir(s).), J. BACHA, S. BEN ABID-ZARROUK, L. KADI, \& A. MABROUR, « Penser les TIC dans les universités du Maghreb », Paris : L’Harmattan, 2016, Pp. 265-283.

OUHAIBIA, B., et al., «Réflexions générales sur l'intégration des TIC dans l'enseignement supérieur algérien », dans muriel frisch (dir(s).), J. BACHA, S. BEN ABID-ZARROUK, L. KADI, \& A. MABROUR, « Penser les TIC dans les universités du Maghreb », Paris : L'Harmattan, 2016, Pp. 219-235.

UNESCO, « Principes directeurs de l'UNESCO pour l'apprentissage mobile », Paris : UNESCO, 2013, $41 \mathrm{P}$.

PRENSKY, M., « Digital Natives, Digital immigrants », On the Horizon, 9, United Kingdom, 2001, Pp1-6.

VENKATESH, V., THONG, Y. L., \& XU, X., « Unified Theory of Acceptance and Use of Technologie : A Synthesis ans the Roas Ahead ", Journal of the Association for Information Systems, 17(5), 2016, Pp. 328-376.

FIÉVEZ, A., \& DUMOUCHEL, G., « Le BYOD : entre perspectives et réalités pédagogiques », 2015, Disponible sur [Ecole branchée : https://ecolebranchee.com/dossier-le-byod-entre-perspectiveset-realites-pedagogiques/], (consulté le 11 août 2019).

LE VINCENT, A.-S., \& BOTINO, M., « BYOD : quand une réalité sociale et économique s'impose au monde de l'éducation », 2017, Disponible sur [https://www.reseau-canope.fr/savoirscdi/cdi-outilpedagogique/conduire-des-projets-realiser-des-activites-pedagogiques/activitespluridisciplinaires/byod-et-contexte-scolaire/byod-quand-une-realite-sociale-et-economiquesimpose-au-monde-de-leducation.html], (consulté le 12 août 2019).

ROUX BELLICAUD, D., « Préparer son premier projet d'utilisation des BYOD en classe », 2014,

Disponible sur [educavox : https://www.educavox.fr/innovation/technologie/preparer-sonpremier-projet-d-utilisation-des-byod-en-classe], (consulté le 10 août 2019).

\section{RÉSUMÉS}

L'apprentissage mobile (ou nomade) est une approche nouvelle faisant appel aux technologies mobiles dans l'enseignement/apprentissage. La présente contribution vise à lever le voile sur cette notion, et à traiter des avis, des représentations et des usages qu'en ont/font des enseignants universitaires algériens. Une enquête par questionnaire a donc été faite pour la récolte de ces différentes données. Les résultats de l'analyse montrent une majorité d'enseignants encline à la mise en œuvre d'une telle démarche, avec le smartphone comme outil de prédilection.

The mobile (or nomadic) learning is a new approach using mobile technologies in teaching/ learning. The present contribution aims shed light on this notion, and to deal with the opinions, representations and uses that are made of it by Algerian university teachers. A questionnaire 
survey was therefore carried out to collect these various data. The analysis results show that a majority of teachers are inclined to implement this approach, with the smartphone as the preferred tool.

INDEX

Keywords : mobile learning, uses, representations, ict, algerian university

Mots-clés : apprentissage mobile, usages, représentations, tic, université algérienne

\section{AUTEURS}

\section{SAMI REDJIMI}

Laboratoire Interdisciplinaire de pédagogie et didactique (LIPED), Université Badji Mokhtar-Annaba, Algérie

\section{BILLEL OUHAIBIA}

Laboratoire Interdisciplinaire de pédagogie et didactique (LIPED), Université Badji MokhtarAnnaba, Algérie 